MATHEMATICS OF COMPUTATION

Volume 69, Number 230, Pages 851-859

S 0025-5718(99)01155-2

Article electronically published on May 24, 1999

\title{
A PARAMETRIC FAMILY OF QUINTIC THUE EQUATIONS
}

\author{
ISTVÁN GAÁL AND GÜNTER LETTL
}

\begin{abstract}
For an integral parameter $t \in \mathbb{Z}$ we investigate the family of Thue equations

$$
\begin{aligned}
F(x, y) & =x^{5}+(t-1)^{2} x^{4} y-\left(2 t^{3}+4 t+4\right) x^{3} y^{2} \\
+ & \left(t^{4}+t^{3}+2 t^{2}+4 t-3\right) x^{2} y^{3}+\left(t^{3}+t^{2}+5 t+3\right) x y^{4}+y^{5}= \pm 1,
\end{aligned}
$$

originating from Emma Lehmer's family of quintic fields, and show that for $|t| \geq 3.28 \cdot 10^{15}$ the only solutions are the trivial ones with $x=0$ or $y=0$. Our arguments contain some new ideas in comparison with the standard methods for Thue families, which gives this family a special interest.
\end{abstract}

\section{INTRODUCTION}

For $t \in \mathbb{Z}$ let us define the polynomial

$$
\begin{aligned}
f_{t}(x)=x^{5}+(t-1)^{2} x^{4}- & \left(2 t^{3}+4 t+4\right) x^{3} \\
+ & \left(t^{4}+t^{3}+2 t^{2}+4 t-3\right) x^{2}+\left(t^{3}+t^{2}+5 t+3\right) x+1 .
\end{aligned}
$$

This family of quintic polynomials was first considered by Emma Lehmer (cf. [6]). Note that instead of the original parameter $n$ we use the parameter $t=n+1$, which fits our arguments better (cf. [11, p. 548]). The corresponding parametric family of totally real cyclic quintic fields $K_{t}=\mathbb{Q}\left(\vartheta_{t}\right), \vartheta_{t}$ a root of $f_{t}$, was also investigated by Schoof and Washington [11] and Darmon [2] for prime conductors $m_{0}=t^{4}+t^{3}+6 t^{2}+6 t+11$.

In a recent paper Gaál and Pohst 3 showed that any four distinct roots of $f_{t}$ form a fundamental system of units in $K_{t}$ for any conductor $m_{0}$, constructed explicitly an integral basis of $K_{t}$ for those $m_{0}$ that are square free apart from 5 , and studied the problem of power integral bases.

In the present paper we will investigate the corresponding family of quintic Thue equations

$$
\begin{aligned}
F(x, y)= & x^{5}+(t-1)^{2} x^{4} y-\left(2 t^{3}+4 t+4\right) x^{3} y^{2} \\
& +\left(t^{4}+t^{3}+2 t^{2}+4 t-3\right) x^{2} y^{3}+\left(t^{3}+t^{2}+5 t+3\right) x y^{4}+y^{5}= \pm 1 .
\end{aligned}
$$

The first infinite parametric family of Thue equations was a cubic one, studied by Thomas [12. He proved that if the parameter is large enough, the equation has only the trivial solutions. His ideas were extended to several other families of cubic

Received by the editor December 12, 1997 and, in revised form, July 14, 1998.

1991 Mathematics Subject Classification. Primary 11D57; Secondary $11 Y 50$.

Key words and phrases. Parametric Thue equation, Baker's method.

The first author's research was supported in part by Grants 16791 and 16975 from the Hungarian National Foundation for Scientific Research. 
and quartic Thue equations in Thomas [13], Pethő 9], Mignotte, Pethö and Roth [8], Lettl and Pethő [7], Pethő and Tichy [10]; for a survey see [5]. Heuberger [4] was the first who obtained results on a quintic family.

The above quoted papers usually apply some standard techniques for Thue equations and Baker's method to derive an upper bound for the unknown exponents of the fundamental units (corresponding to a non-trivial solution) in terms of the parameter. If the rank of the unit group is small, a difference in the size of the logarithms of the fundamental units implies a difference in the order of magnitude of the exponents. This allows us to derive a lower bound for the largest exponent, contradicting the upper bound and thus proving the non-existence of non-trivial solutions if the parameter is large enough.

Investigating equation (1), we have to handle a unit group of rank 4, and it turns out that the exponents have the same order of magnitude. So we had to design a new method to overcome this problem. Using asymptotic expansions of the expressions involved, we construct non-vanishing linear combinations of the exponents, which are small. These allow us to derive lower bounds for $|y|$ and the largest exponent, and again one obtains the desired contradiction for sufficiently large parameters. This method can be applied for families of Thue equations with high unit rank, as long as the coefficients of the asymptotic expansions of the roots with respect to the parameter are rational.

The main result of this paper is the following.

Theorem. For $|t| \geq 3.28 \cdot 10^{15}$ the only integral solutions of equation (1) are the trivial ones,

$$
(x, y)=( \pm 1,0),(0, \pm 1) .
$$

We remark that for the calculations involved in our estimates we extensively used Maple.

\section{Elementary estimates}

For $t \in \mathbb{Z}$, let $\alpha$ be a root of the polynomial $f_{t}(x)=F(x, 1)$. The field $K=$ $\mathbb{Q}(\alpha)$ is a totally real cyclic field, the Galois group of which is generated by the automorphism

$$
\varphi: \alpha \longmapsto \alpha^{\prime}=\frac{(t+1)+(t-1) \alpha-\alpha^{2}}{1+(t+1) \alpha} .
$$

We denote the roots of $f_{t}(x)$ by $\alpha_{1}, \ldots, \alpha_{5}$, such that $\alpha_{1}$ is the smallest one and $\varphi\left(\alpha_{i}\right)=\alpha_{i+1}$ for $i=1, \ldots, 5$ (note that here and in the following the indices of the $\alpha_{i}$ are to be taken $\left.\bmod (5)\right)$. According to [3], any four distinct roots of $f_{t}(x)$ form a system of fundamental units in $K$.

From the asymptotic expansions of the roots we obtain that for $|t| \geq 100$ one has

$$
\begin{gathered}
\alpha_{1}=-t^{2}-2-\frac{1+\delta_{1}}{t}, \quad \alpha_{2}=t+\frac{1}{t}-\frac{1}{t^{3}}-\frac{1+\delta_{2}}{t^{4}} \\
\alpha_{3}=-\frac{1}{t^{3}}+\frac{1}{t^{4}}+\frac{3}{t^{5}}-\frac{4+\delta_{3}}{t^{6}}, \quad \alpha_{4}=t+1+\frac{1}{t}-\frac{1}{t^{3}}-\frac{1+\delta_{4}}{t^{4}} \\
\alpha_{5}=-\frac{1}{t}+\frac{1+\delta_{5}}{t^{4}},
\end{gathered}
$$


with some $\left|\delta_{i}\right|<0.1(i=1, \ldots, 5)$. This can be checked by substituting these expressions with $\delta_{i}= \pm 0.1$ into $f_{t}(x)$ and observing that $f_{t}(x)$ changes its sign between these two arguments. Let us remark that for $t \geq 0$ one can show that $\alpha_{1}<\alpha_{5}<\alpha_{3}<\alpha_{2}<\alpha_{4}$, whereas for $t<0$ one has $\alpha_{1}<\alpha_{2}<\alpha_{4}<\alpha_{3}<\alpha_{5}$.

If $y=0$, (1) has only the trivial solutions $( \pm 1,0)$. So let us assume for the following that $|t| \geq 100$ and that $(x, y) \in \mathbb{Z}^{2}$ is a solution of (1) with $|y| \geq 1$. Put

$$
\beta_{j}=x-\alpha_{j} y \quad \text { for } j=1, \ldots 5,
$$

and define $i$ to be that index with $\left|\beta_{i}\right|=\min _{1 \leq j \leq 5}\left|\beta_{j}\right|$. Then for $m \neq i$ we obtain

$$
\left|\alpha_{m}-\frac{x}{y}\right|<\left|\alpha_{m}-\tilde{\alpha}_{i, m}\right| \text {, }
$$

where $\tilde{\alpha}_{i, m}$ is the arithmetic mean of $\alpha_{i}$ and its neighbouring root between $\alpha_{i}$ and $\alpha_{m}$. (This may be different for $t \geq 0$ and $t<0$. Indeed, if e.g. $i=4$ and $t \geq 0$ then $\tilde{\alpha}_{4, m}=\left(\alpha_{2}+\alpha_{4}\right) / 2$ for all $m \neq 4$, whereas for $t<0$ one has $\tilde{\alpha}_{4,1}=\tilde{\alpha}_{4,2}=\left(\alpha_{2}+\alpha_{4}\right) / 2$ and $\tilde{\alpha}_{4,3}=\tilde{\alpha}_{4,5}=\left(\alpha_{3}+\alpha_{4}\right) / 2$.) Using (3), we deduce that

$$
\left|\alpha_{i}-\frac{x}{y}\right|=\frac{1}{|y|^{5}} \cdot \frac{1}{\prod_{m \neq i}\left|\alpha_{m}-\frac{x}{y}\right|} \leq \frac{1}{|y|^{5}} \cdot \frac{1}{\prod_{m \neq i}\left|\alpha_{m}-\tilde{\alpha}_{i, m}\right|},
$$

and by estimates (2) for the roots we obtain, for all $i=1, \ldots, 5$,

$$
\left|\alpha_{i}-\frac{x}{y}\right|<\frac{8.06}{|y|^{5}|t|^{3}} \text {. }
$$

Since $|t| \geq 100$, this implies $\left|\alpha_{i}-x / y\right|<1 /\left(2|y|^{2}\right)$; thus $x / y$ is a convergent arising from the continued fraction expansion of $\alpha_{i}$. Calculating the continued fraction expansion of the roots, we obtain for $t \geq 5$

$$
\begin{aligned}
& \alpha_{1}=\left\langle-t^{2}-3 ; 1, t-1,[t / 3], \ldots\right\rangle, \\
& \alpha_{2}=\langle t ; t, t-1, \ldots\rangle, \\
& \alpha_{3}=\left\langle-1 ; 1, t^{3}+t^{2}+4 t+2, \ldots\right\rangle, \\
& \alpha_{4}=\langle t+1 ; t, t-1, \ldots\rangle, \\
& \alpha_{5}=\left\langle-1 ; 1, t-1, t^{2}-t+4, \ldots\right\rangle,
\end{aligned}
$$

and for $t \leq-8$

$$
\begin{aligned}
& \alpha_{1}=\left\langle-t^{2}-2 ;|t|,[(|t|-1) / 3], \ldots\right\rangle, \\
& \alpha_{2}=\langle t-1 ; 1,|t|-1,|t|+1, \ldots\rangle, \\
& \alpha_{3}=\left\langle 0 ;-t^{3}-t^{2}-4 t-3,[|t| / 2]-1, \ldots\right\rangle, \\
& \alpha_{4}=\langle t ; 1,|t|-1,|t|+1, \ldots\rangle, \\
& \alpha_{5}=\left\langle 0 ;|t|-1,1, t^{2}+|t|+4, \ldots\right\rangle,
\end{aligned}
$$

where $[x]$ denotes the largest integer not exceeding $x$. To get the continued fraction expansion of the roots we had Maple calculate them for $-20 \leq t \leq 20$, from which we guessed the general shape of the partial quotients. For the proof of these expansions let us note that the partial quotients $a_{j}$ for all $0 \leq j \leq m$ of the continued fraction expansion of some real number $\alpha=\left[a_{0} ; a_{1}, a_{2}, \ldots\right]$ are determined by the property that $\alpha$ lies between $\left[a_{0} ; a_{1}, \ldots, a_{m}\right]$ and $\left[a_{0} ; a_{1}, \ldots, a_{m}+1\right]$. Calculating the above expressions with (an upper bound for) the last indicated partial quotient 
$a_{m}$ and (a lower bound for) $a_{m}+1$, resp., one verifies that $f_{t}(x)$ changes its sign between these two values for all $t$ which are to be considered.

From the above continued fractions we can calculate all convergents of the roots with denominators less than $t^{2}+1$ (in the case of $\alpha_{1}$ one also has to consider some possible small values for the next two partial quotients which are not indicated in the above expansions). Substituting the numerators and denominators of these convergents for $x, y$ into (1), we do not get any solutions except the trivial one $(0, \pm 1)$. For this reason we conclude that

$$
|y|>t^{2} .
$$

\section{BAKER'S METHOD}

Let $j, k$ be such that $i, j, k$ are distinct indices among $1, \ldots, 5$. From Siegel's identity

$$
\left(\alpha_{i}-\alpha_{j}\right) \beta_{k}+\left(\alpha_{j}-\alpha_{k}\right) \beta_{i}+\left(\alpha_{k}-\alpha_{i}\right) \beta_{j}=0
$$

and using (4), we obtain

$$
\begin{aligned}
|| \frac{\alpha_{i}-\alpha_{j}}{\alpha_{i}-\alpha_{k}} \cdot \frac{\beta_{k}}{\beta_{j}}|-1| \leq\left|\frac{\alpha_{i}-\alpha_{j}}{\alpha_{i}-\alpha_{k}} \cdot \frac{\beta_{k}}{\beta_{j}}-1\right|=\left|\frac{\alpha_{k}-\alpha_{j}}{\alpha_{i}-\alpha_{k}} \cdot \frac{\beta_{i}}{\beta_{j}}\right| \\
\leq\left|\frac{\alpha_{k}-\alpha_{j}}{\alpha_{i}-\alpha_{k}}\right| \cdot\left|\frac{\alpha_{i}-\frac{x}{y}}{\alpha_{j}-\frac{x}{y}}\right|<\left|\frac{\alpha_{k}-\alpha_{j}}{\alpha_{i}-\alpha_{k}}\right| \cdot \frac{8.06|y|^{-5}|t|^{-3}}{\left|\alpha_{j}-\alpha_{i}\right|-8.06|t|^{-3}} .
\end{aligned}
$$

In order to get appropriate estimates we choose $(i, j, k)=(1,3,5),(2,5,3),(3,1,4)$, $(4,1,3),(5,2,4)$ in the five cases that are to be considered, depending on the value of $i$. By (2) one obtains that for all $t$ with $|t| \geq 100$

$$
\left|\frac{\alpha_{k}-\alpha_{j}}{\alpha_{i}-\alpha_{k}}\right| \cdot \frac{8.06|y|^{-5}|t|^{-3}}{\left|\alpha_{j}-\alpha_{i}\right|-8.06|t|^{-3}}<\frac{8.1}{|y|^{5}|t|^{4}} .
$$

Since for any real $z \geq 0.2032$ the inequality $|\log (z)|<2|z-1|$ holds, we finally conclude from these estimates that for each $i \in\{1, \ldots, 5\}$

$$
\Lambda_{i}:=|\log | \frac{\alpha_{i}-\alpha_{j}}{\alpha_{i}-\alpha_{k}} \cdot \frac{\beta_{k}}{\beta_{j}}|| \leq 2|| \frac{\alpha_{i}-\alpha_{j}}{\alpha_{i}-\alpha_{k}} \cdot \frac{\beta_{k}}{\beta_{j}}|-1|<\frac{16.2}{|y|^{5}|t|^{4}} .
$$

Next we are going to apply Baker's method to get a lower estimate for $\Lambda_{i}$. We take $\alpha_{1}, \alpha_{2}, \alpha_{3}, \alpha_{4}$ as a system of fundamental units. Then there are $u_{1}, \ldots, u_{4} \in \mathbb{Z}$ such that for $m=1, \ldots, 5$ we have

$$
\beta_{m}=\alpha_{m}^{u_{1}} \alpha_{m+1}^{u_{2}} \alpha_{m+2}^{u_{3}} \alpha_{m+3}^{u_{4}},
$$

and so

$$
\Lambda_{i}=|\log | \frac{\alpha_{i}-\alpha_{j}}{\alpha_{i}-\alpha_{k}}\left|+u_{1} \log \right| \frac{\alpha_{k}}{\alpha_{j}}\left|+u_{2} \log \right| \frac{\alpha_{k+1}}{\alpha_{j+1}}\left|+u_{3} \log \right| \frac{\alpha_{k+2}}{\alpha_{j+2}}\left|+u_{4} \log \right| \frac{\alpha_{k+3}}{\alpha_{j+3}}|| .
$$

To obtain good estimates for $\log \left|\alpha_{m}\right|$ and $\log \left|\alpha_{m}-\alpha_{n}\right|$ we derive from the series expansion of the logarithm the following auxiliar result:

Lemma. Let $a_{1}, a_{2}, a_{3}, t \in \mathbb{R}$ with $|t| \geq 100,\left|a_{1}\right| \leq 1,\left|a_{2}\right| \leq 3,\left|a_{3}\right| \leq 5$ and $-3<-a_{1} a_{3}+a_{1}^{2} a_{2}<8$. Then

$$
\log \left(1+\frac{a_{1}}{t}+\frac{a_{2}}{t^{2}}+\frac{a_{3}}{t^{3}}\right)=\frac{a_{1}}{t}+\frac{a_{2}-a_{1}^{2} / 2}{t^{2}}+\frac{a_{3}-a_{1} a_{2}+a_{1}^{3} / 3+\tilde{\delta}}{t^{3}}
$$

for some $\tilde{\delta} \in \mathbb{R}$ with $|\tilde{\delta}|<0.1$. 
Proof. Putting $c:=a_{1} / t+a_{2} / t^{2}+a_{3} / t^{3}$, the above conditions yield $|c| \leq 1.0305 /|t|$, and by estimating the series expansion of the logarithm we obtain

$$
0 \leq c-\frac{c^{2}}{2}+\frac{c^{3}}{3}-\log (1+c)<\frac{0.003}{|t|^{3}} .
$$

Next one can show that the difference between $c-c^{2} / 2+c^{3} / 3$ and the expression claimed in the lemma is at most $0.083 /|t|^{3}$. Combining these inequalities, we get the assertion.

Applying our lemma and using (2), we obtain the following estimates:

$$
\begin{aligned}
& \log \left|\alpha_{1}\right|=2 \log |t|+\frac{2}{t^{2}}+\frac{1+\delta_{6}}{t^{3}} \\
& \log \left|\alpha_{2}\right|=\log |t|+\frac{1}{t^{2}}+\frac{\delta_{7}}{t^{3}} \\
& \log \left|\alpha_{3}\right|=-3 \log |t|-\frac{1}{t}-\frac{7}{2 t^{2}}+\frac{2 / 3+\delta_{8}}{t^{3}}, \\
& \log \left|\alpha_{4}\right|=\log |t|+\frac{1}{t}+\frac{1}{2 t^{2}}+\frac{-2 / 3+\delta_{9}}{t^{3}} \\
& \log \left|\alpha_{5}\right|=-\log |t|+\frac{-1+\delta_{10}}{t^{3}}
\end{aligned}
$$

where $\left|\delta_{m}\right|<0.2, m=6, \ldots, 10$, and similarly,

$$
\begin{aligned}
& \log \left|\alpha_{1}-\alpha_{2}\right|=2 \log |t|+\frac{1}{t}+\frac{3}{2 t^{2}}+\frac{1 / 3+\delta_{11}}{t^{3}} \\
& \log \left|\alpha_{1}-\alpha_{3}\right|=2 \log |t|+\frac{2}{t^{2}}+\frac{1+\delta_{12}}{t^{3}} \\
& \log \left|\alpha_{1}-\alpha_{4}\right|=2 \log |t|+\frac{1}{t}+\frac{5}{2 t^{2}}+\frac{-2 / 3+\delta_{13}}{t^{3}} \\
& \log \left|\alpha_{1}-\alpha_{5}\right|=2 \log |t|+\frac{2}{t^{2}}+\frac{\delta_{14}}{t^{3}} \\
& \log \left|\alpha_{2}-\alpha_{3}\right|=\log |t|+\frac{1}{t^{2}}+\frac{\delta_{15}}{t^{3}} \\
& \log \left|\alpha_{2}-\alpha_{4}\right|=\frac{\delta_{16}}{t^{3}}, \\
& \log \left|\alpha_{2}-\alpha_{5}\right|=\log |t|+\frac{2}{t^{2}}+\frac{\delta_{17}}{t^{3}} \\
& \log \left|\alpha_{3}-\alpha_{4}\right|=\log |t|+\frac{1}{t}+\frac{1}{2 t^{2}}+\frac{-2 / 3+\delta_{18}}{t^{3}} \\
& \log \left|\alpha_{3}-\alpha_{5}\right|=-\log |t|-\frac{1}{t^{2}}+\frac{\delta_{19}}{t^{3}}, \\
& \log \left|\alpha_{4}-\alpha_{5}\right|=\log |t|+\frac{1}{t}+\frac{3}{2 t^{2}}+\frac{-5 / 3+\delta_{20}}{t^{3}}
\end{aligned}
$$

where $\left|\delta_{m}\right|<0.3, m=11, \ldots, 20$.

Now we apply the theorem of Baker and Wüstholz [1, p. 20] to the linear form of logarithms (8) with $n=d=5$ and $U=\max \left\{\left|u_{1}\right|, \ldots,\left|u_{4}\right|\right\}$. This yields

$$
\log \left|\Lambda_{i}\right|>-18 \cdot 6 ! 5^{6} 160^{7} \log 50 \cdot h^{\prime}\left(\frac{\alpha_{i}-\alpha_{j}}{\alpha_{i}-\alpha_{k}}\right) h^{\prime}\left(\frac{\alpha_{k}}{\alpha_{j}}\right)^{4} \log U .
$$


Note that the terms of the linear forms are linearly independent over $\mathbb{Q}$, since by our choice of the indices $(i, j, k)$ the numbers $\left(\alpha_{i}-\alpha_{j}\right) /\left(\alpha_{i}-\alpha_{k}\right)$ are not units. Using (9) and (10), it is a routine matter to estimate the heights of the algebraic numbers involved, and we obtained

$$
h^{\prime}\left(\frac{\alpha_{k}}{\alpha_{j}}\right)=h^{\prime}\left(\frac{\alpha_{1}}{\alpha_{3}}\right)<1.0005 \log |t| \quad \text { and } \quad h^{\prime}\left(\frac{\alpha_{i}-\alpha_{j}}{\alpha_{i}-\alpha_{k}}\right)<0.801 \log |t|,
$$

and finally the lower bound

$$
\Lambda_{i}>\exp \left(-0.1707 \cdot 10^{25}(\log |t|)^{5} \log U\right),
$$

valid for each $i$.

\section{A LOWER BOUND FOR $U$}

Taking logarithms of the absolute values of (7) yields the system of equations

$$
\log |y|+\log \left|\alpha_{m}-\frac{x}{y}\right|=u_{1} \log \left|\alpha_{m}\right|+u_{2} \log \left|\alpha_{m+1}\right|+u_{3} \log \left|\alpha_{m+2}\right|+u_{4} \log \left|\alpha_{m+3}\right|
$$

for $m=1, \ldots, 5, \quad m \neq i$. The determinant of this system of linear equations in $u_{1}, \ldots, u_{4}$ is, up to sign, just the regulator $R_{K}$ of the field $K$, and using (9) we get

$$
\begin{aligned}
R_{K} & =71(\log |t|)^{4}+\frac{56(\log |t|)^{3}}{t}+\frac{308(\log |t|)^{3}+21(\log |t|)^{2}+\delta_{21}}{t^{2}} \\
& =\left(71+\delta_{22}\right)(\log |t|)^{4}
\end{aligned}
$$

with $\left|\delta_{21}\right|<115$ and $\left|\delta_{22}\right|<0.13$

Note that by (4) and (5) we have

$$
\log \left|\alpha_{m}-\frac{x}{y}\right|=\log \left|\alpha_{m}-\alpha_{i}+\frac{\delta_{23}}{t^{13}}\right|
$$

where $\left|\delta_{23}\right|<8.06$. By $(2)$ we obtain for these expressions the same asymptotic expansions as in (10) for $\left|\alpha_{m}-\alpha_{i}\right|$, again with some $\left|\delta_{m}\right|<0.3$ for $m=11, \ldots, 20$.

For each case of $i \in\{1, \ldots, 5\}$ we solve the system of linear equations (12) by using Cramer's rule and obtain

$$
\begin{aligned}
R_{K} u_{j}= & \log |y|\left(d_{j 1}(\log |t|)^{3}+\frac{d_{j 2}(\log |t|)^{2}}{t}+\frac{d_{j 3}(\log |t|)^{2}+d_{j 4} \log |t|+d_{j 5}}{t^{2}}\right) \\
& +e_{j 1}(\log |t|)^{4}+\frac{e_{j 2}(\log |t|)^{3}}{t}+\frac{e_{j 3}(\log |t|)^{3}+e_{j 4}(\log |t|)^{2}+e_{j 5}}{t^{2}}
\end{aligned}
$$

for $j=1, \ldots, 4$, with rational coefficients $d_{j k}, e_{j k} \in \mathbb{Q}$ for $1 \leq k \leq 4$ and bounds for $d_{j 5}, e_{j 5}$. It turns out that in all cases the $u_{j}$ 's have the same order of magnitude. Therefore we look for integral linear combinations $b_{0} R_{K}+b_{1} R_{K} u_{1}+\cdots+b_{4} R_{K} u_{4}$ with $b_{j} \in \mathbb{Z}$ which are positive and small in such a way that the coefficients of the main terms $\log |y|(\log |t|)^{3}, \log |y|(\log |t|)^{2} / t,(\log |t|)^{4}$ and $(\log |t|)^{3} / t$ vanish. This amounts to solving a system of linear Diophantine equations. Then since

$$
1 \leq v=b_{0}+b_{1} u_{1}+\cdots+b_{4} u_{4} \in \mathbb{Z}
$$


and

(16)

$$
R_{K} v=\log |y|\left(\frac{d_{6}(\log |t|)^{2}+d_{7} \log |t|+d_{8}}{t^{2}}\right)+\frac{e_{6}(\log |t|)^{3}+e_{7}(\log |t|)^{2}+e_{8}}{t^{2}},
$$

we can use $R_{K} v \geq R_{K}$ and (13) to derive a lower bound for $\log |y|$ of the form

$$
\log |y|>c_{1} t^{2}(\log |t|)^{2} .
$$

Explicitly, in the five cases for $i$ we arrived at the following values for the $b_{j}$ 's in (15):

\begin{tabular}{c|c|c|c|c|c}
$i$ & $b_{0}$ & $b_{1}$ & $b_{2}$ & $b_{3}$ & $b_{4}$ \\
\hline 1 & 0 & 10 & 3 & 9 & -27 \\
\hline 2 & -22 & -65 & 49 & -51 & -15 \\
\hline 3 & 1 & -1 & -21 & 10 & -8 \\
\hline 4 & 6 & -6 & -5 & 18 & -17 \\
\hline 5 & 1 & -5 & 18 & -17 & 10
\end{tabular}

and for the coefficients of $R_{K} v$ in (16) and the constant $c_{1}$ in (17) we obtained

\begin{tabular}{c|c|c|c|c|c|c|c}
$i$ & $d_{6}$ & $d_{7}$ & $\left|d_{8}\right|<$ & $e_{6}$ & $e_{7}$ & $\left|e_{8}\right|<$ & $c_{1}$ \\
\hline 1 & 710 & 170 & 136 & 1349 & 269 & 1860 & 0.094 \\
\hline 2 & 2130 & 130 & 591 & 2769 & 363 & 4268 & 0.032 \\
\hline 3 & 355 & 180 & 52 & 71 & 0 & 498 & 0.178 \\
\hline 4 & 355 & -10 & 114 & 284 & 3 & 989 & 0.196 \\
\hline 5 & 355 & -10 & 114 & -71 & -1 & 670 & 0.197
\end{tabular}

From $d_{6}, e_{6}>0$ in the cases $1 \leq i \leq 4$ we immediately have $R_{K} v>0$; thus $v \geq 1$. For $i=5$ we used $\log |y|>2 \log |t|$ from (5) to show the same.

To improve the value of $c_{1}$ in the cases $i=1,2$ we looked for other $b_{j}$ 's giving a smaller coefficient $d_{6}$ for $R_{K} v$, but no longer requiring the term $(\log |t|)^{3} / t$ to vanish, and found

\begin{tabular}{c|c|c|c|c|c|c}
$i$ & $b_{0}$ & $b_{1}$ & $b_{2}$ & $b_{3}$ & $b_{4}$ & $c_{1}$ \\
\hline 1 & 0 & -26 & 37 & -11 & -15 & 0.319 \\
\hline 2 & -3 & -17 & 10 & -6 & -5 & 0.197
\end{tabular}

With the lower bound (17) for $\log |y|$ obtained in the first step we could show that again $R_{K} v>0$, and so we get the improved values for $c_{1}$ as indicated in the above table.

From the lower bound (17) for $\log |y|$ it is clear that $U=\max _{1 \leq n \leq 4}\left|u_{n}\right|=\left|u_{j}\right|$ for that $u_{j}$ having the largest coefficient $d_{j 1}$ (in absolute value) in its expansion 
(14). Using this $u_{j},(13)$ and (17), we have

$$
\begin{aligned}
\left(71+\delta_{22}\right)(\log |t|)^{4} U= & R_{K}\left|u_{j}\right| \\
>c_{1} t^{2}(\log |t|)^{2} & \left(\left|d_{j 1}\right|(\log |t|)^{3}-\frac{\left|d_{j 2}\right|(\log |t|)^{2}}{|t|}\right. \\
& \left.-\frac{\left|d_{j 3}\right|(\log |t|)^{2}+\left|d_{j 4}\right|(\log |t|)+\left|d_{j 5}\right|}{t^{2}}\right) \\
-\left|e_{j 1}\right|(\log |t|)^{4} & -\frac{\left|e_{j 2}\right|(\log |t|)^{3}}{|t|}-\frac{\left|e_{j 3}\right|(\log |t|)^{3}+\left|e_{j 4}\right|(\log |t|)+\left|e_{j 5}\right|}{t^{2}},
\end{aligned}
$$

which gives a lower bound for $U$ of the type

$$
U>c_{2} t^{2} \log |t|
$$

with $0.1548 \leq c_{2} \leq 0.285$, depending on the different cases of $i$.

On the other hand, the inequality

$$
\begin{aligned}
(71+ & \left.\delta_{22}\right)(\log |t|)^{4} U=R_{K}\left|u_{j}\right| \\
< & \log |y|\left(\left|d_{j 1}\right|(\log |t|)^{3}+\frac{\left|d_{j 2}\right|(\log |t|)^{2}}{|t|}+\frac{\left|d_{j 3}\right|(\log |t|)^{2}+\left|d_{j 4}\right| \log |t|+\left|d_{j 5}\right|}{t^{2}}\right) \\
& +\left|e_{j 1}\right|(\log |t|)^{4}+\frac{\left|e_{j 2}\right|(\log |t|)^{3}}{|t|}+\frac{\left|e_{j 3}\right|(\log |t|)^{3}+\left|e_{j 4}\right| \log |t|+\left|e_{j 5}\right|}{t^{2}}
\end{aligned}
$$

yields

$$
c_{3} U \log |t|<\log |y|
$$

with $0.686<c_{3}<1.358$. Combining (6) with (19), we conclude that

$$
\Lambda_{i}<\exp (2.8-3.43 U \log |t|-4 \log |t|)<\exp (-3.43 U \log |t|) .
$$

This upper bound together with the lower estimate (11) yields

$$
\frac{U}{\log U}<4.98 \cdot 10^{23}(\log |t|)^{4} .
$$

Using (18), by the monotony of $x / \log x$ we get

$$
\frac{0.1548 t^{2} \log |t|}{\log \left(0.1548 t^{2} \log |t|\right)}<4.98 \cdot 10^{23}(\log |t|)^{4} .
$$

This inequality holds for $|t|=3.27 \cdot 10^{15}$ but fails for $|t|=3.28 \cdot 10^{15}$, which implies that the existence of a non-trivial solution leads to a contradiction if

$$
|t| \geq 3.28 \cdot 10^{15} \text {. }
$$

\section{REFERENCES}

1. A. Baker \& G. Wüstholz, Logarithmic forms and group varieties, J. Reine Angew. Math. 442 (1993), 19-62. MR 94i:11050]

2. H. Darmon, Note on a polynomial of Emma Lehmer, Math. Comp. 56 (1991), 795-800. MR 91i:11149

3. I. Gaál \& M. Pohst, Power integral bases in a parametric family of totally real cyclic quintics, Math. Comp. 66 (1997), 1689-1696. MR 98a:11160

4. C. Heuberger, On a family of quintic Thue equations, J. Symbolic Comput. 26 (1998), 173185. CMP 98:16

5. C. Heuberger, A. Pethő \& R.F. Tichy, Complete solution of parametrized Thue equations, Acta Math. Inform. Univ. Ostraviensis 6 (1998), 93-114. 
6. E. Lehmer, Connection between Gaussian periods and cyclic units, Math. Comp. 50 (1988), 535-541. MR 89h:11067a

7. G. Lettl \& A. Pethö, Complete solution of a family of quartic Thue equations, Abh. Math. Sem. Univ. Hamburg 65 (1995), 365-383. MR 96h:11019

8. M. Mignotte, A. Pethö \& R. Roth, Complete solutions of a family of quartic Thue and index form equations, Math. Comp. 65 (1996), 341-354. MR 96d:11034

9. A. Pethö, Complete solutions to families of quartic Thue equations, Math. Comp. 57 (1991), 777-798. MR 92e:11023

10. A. Pethő \& R.F. Tichy, On two-parametric quartic families of Diophantine problems, J. Symbolic Comput. 26 (1998), 151-171. CMP 98:16

11. R. Schoof \& L. Washington, Quintic polynomials and real cyclotomic fields with large class numbers, Math. Comp. 50 (1988), 543-556. MR 89h:11067b

12. E. Thomas, Complete solutions to a family of cubic diophantine equations, J. Number Theory 34 (1990), 235-250. MR 91b:11027

13. E. Thomas, Solutions to certain families of Thue equations, J. Number Theory 43 (1993), 319-369. MR 94b:11028

Kossuth Lajos University, Mathematical Institute, H-4010 Debrecen Pf.12., Hungary E-mail address: igaal@math.klte.hu

Karl-Franzens-Universität Graz, Institut für Mathematik, A-8010 Graz, HeinrichStrasse 36, Austria

E-mail address: guenter.lettl@kfunigraz.ac.at 